\title{
„Wo bleibt der demokratische Geschichtsunterricht?" Der Umgang mit der NS-Vergangenheit in Schulbildung und Gesellschaft am Beispiel von Taras Borodajkewycz und Hans-Ulrich Rudel
}

\author{
Benedikt Kapferer \\ Kerngebiet: Zeitgeschichte \\ eingereicht bei: Dr. Karin Moser \\ eingereicht im: SoSe 2019 \\ Rubrik: Varia
}

\begin{abstract}
"Where are the democratic history lessons?" Dealing with the Nazi past in education and society in Austria with the examples of Taras Borodajkewycz and Hans-Ulrich Rudel

In post-WWII Austria, the way the Nazi past was dealt with was far from frictionless or consensual. As opposed to the preceding ideologies of Fascism and National Socialism, a new democratic mentality had yet to be formed. In this regard, history lessons at universities and at schools are central spaces for analysing the processes of de-Nazification and democratization. Therefore, the following paper discusses two examples of highly controversial teachings that reflect the larger level of Austrian history after 1945: Taras Borodajkewycz (1960s) and Hans-Ulrich Rudel (1983).
\end{abstract}

\section{Einleitung}

„Die einzig wahrhafte Kraft gegen das Prinzip von Auschwitz wäre Autonomie [...]; die Kraft zur Reflexion, zur Selbstbestimmung, zum Nicht-Mitmachen." 
Mit seinem mittlerweile zum Klassiker gewordenen Text „Erziehung nach Auschwitz” aus dem Jahr 1966 prägte Theodor W. Adorno den gesellschaftlichen Zugang zum Umgang mit der NS-Vergangenheit auf einzigartige Weise. So wurden die darin aufgestellten Grundsätze über Erziehung zu einem „Credo“ für eine ganze Generation von Lehrer*innen. ${ }^{2}$ Damit steht er insbesondere in der Bundesrepublik Deutschland für eine tiefgreifende Veränderung im (Geschichts-)Bewusstsein der Nachkriegszeit. Doch gerade in den ersten Jahrzehnten nach dem Zweiten Weltkrieg gab es keineswegs eine allgemeine Deutungshoheit und keinen gesellschaftlichen Konsens darüber, wie mit dem Nationalsozialismus in Erziehung, Schule und in der Geschichtsvermittlung umgegangen werden sollte. Die Bereitschaft zum (selbst-)reflexiven, selbstbestimmten und kritischen Diskurs über die jüngere Vergangenheit war zweifelsfrei nur sehr schwach ausgeprägt, weshalb die Grundlage dafür erst in der öffentlichen Auseinandersetzung gelegt werden musste.

In der Geschichte der Zweiten Republik gilt die Affäre um Taras Borodajkewycz (19021984), Professor für Neuere Geschichte der Hochschule für Welthandel in Wien, als ein zentrales Kapitel für die Aushandlung des Umgangs mit dem Erbe des Nationalsozialismus. Der von linken Studierenden und Aktivist*innen getragene Protest richtete sich gegen den Hochschulprofessor aufgrund seines öffentlich zur Schau gestellten deutschnationalen und österreichfeindlichen Geschichtsbildes, seines größtenteils hingenommenen, unhinterfragten und als belustigend aufgefassten Antisemitismus und seiner Bewertung des Nationalsozialismus und Adolf Hitlers in seiner Lehre. Ende März 1965 kam es zu Demonstrationen und Ausschreitungen zwischen Gegner*innen und Befürworter*innen des Professors, bei denen es mit dem ehemaligen Widerstandskämpfer Ernst Kirchweger das erste politische Todesopfer der Zweiten Republik gab. Die Auseinandersetzungen machten den uneinheitlichen Umgang mit der NS-Vergangenheit in der Gesellschaft sichtbar. Im Speziellen warfen die Proteste ein Licht auf die nicht vorhandene kritische Behandlung des Nationalsozialismus im Rahmen der Geschichtsvermittlung, im konkreten Fall in der universitären Lehre und Hochschulbildung. Als besonders prägnanter Ausdruck dafür steht die Formulierung „Wo bleibt der demokratische Geschichtsunterricht? ${ }^{\text {“3 }}$ von einem Plakat eines Demonstranten aus dem Anti-Borodajkewycz-Lager.

Ausgehend von dieser Parole geht die vorliegende Arbeit der Frage nach, wie sich der Umgang mit der NS-Vergangenheit in der Geschichtsvermittlung in der Nachkriegsgesellschaft der Zweiten Republik gestaltete. Wie wurde Zeitgeschichte als jüngere Vergangenheit thematisiert und in institutionalisierter Form vermittelt? Wie lässt sich der Prozess der Loslösung von autoritären Strukturen und NS-freundlichen Gesinnungen

2 Andrea Höft, Nationalsozialismus im Schulunterricht, in: Torben Fischer/Matthias Lorenz (Hrsg.), Lexikon der „Vergangenheitsbewältigung" in Deutschland. Debatten- und Diskursgeschichte des Nationalsozialismus nach 1945, Berlin 2015, S. 182-186, hier S. 184.

3 Johannes Lau, Langsame Abkehr von der Opferthese, in: Der Standard, 3.10.2018, S. 28; vgl. auch 19650330_ PD0002 (RM), 30.03.1965. APA Picturedesk [https://www.picturedesk.com/bild-disp/search/search.action?ts=1555661039786\&data=eyJicil6WylwOjlxOmJvem9kYWprZXd5Y3oiXSwicnMiOjEsInNOljoiUVVFUIIfU1RSSU5HIiw iaXNwljpmYWxzZSwiaXNzljpmYWxzZSwic29ydCI6MCwibnJwljo1 MCwicG4iOjF9\&searchID=20d2da56-eb134579-8a9b-78290ce13bd8], eingesehen 19.04.2019. 
hin zu einer Demokratisierung der Gesellschaft im Sinne eines "demokratischen Geschichtsunterrichts" verstehen? Zur Behandlung dieser Fragen stehen zwei Fallbeispiele im Mittelpunkt: zum einen die bereits skizzierte Affäre Borodajkewycz und zum anderen die Folge "Geschichtsunterricht" der ORF-Sendung "Ohne Maulkorb" aus dem Jahr 1983. Neben der fehlenden Behandlung der Zeitgeschichte spricht darin ein Schüler einen Vorfall von eines Geschichtelehrers an einer Wiener Schule an. Dieser habe seiner Klasse nahegelegt, sich an Hans-Ulrich Rudel (1916-1982), einem Schlachtflieger der Wehrmacht, NS-Fluchthelfer und später noch aktiven Nationalsozialisten, ein Beispiel zu nehmen und ihn als Helden zu betrachten. Die beiden Fälle dienen als Beispiele dafür, wie der Diskurs über den Nationalsozialismus in der schulischen und universitären Lehre in Österreich bis weit in die Zweite Republik (zumindest) in Teilen geführt wurde. Dabei wird von der These ausgegangen, dass diese öffentlichen Infragestellungen von Praktiken der Geschichtsvermittlung in einem längeren Prozess einer Entnazifizierung von unten und gleichzeitig einer Demokratisierung von unten entsprachen. Im Gegensatz zu politisch „von oben herab“ (top-down) verordneten gesetzlichen Maßnahmen meint die Bezeichnung "von unten" (bottom-up) eine im Wesentlichen aus der Gesellschaft kommende außerparlamentarische Entwicklung. Wenngleich es sich bei den zentralen Akteur*innen um Angehörige einer Bildungselite handelte, steht der Diskurs im Kontrast zur politischen Handlungsebene im engeren Sinne (Gesetze, Verordnungen, parlamentarische Mittel etc.). Eine Entnazifizierung bzw. Demokratisierung von unten betont demnach anstelle von hohen politischen Entscheidungsträger*innen die Rolle der Bevölkerung, der Zivilgesellschaft oder einzelner Individuen sowie vor allem jüngerer Generationen.

Die Entnazifizierung in Österreich wird zwar meist als gescheitert betrachtet, ${ }^{4}$ in den ausgewählten Beispielen ist jedoch zu erkennen, dass vor allem jüngere Generationen immer wieder für eine solche Entnazifizierung eintraten und eine Demokratisierung der politisch-sozialen Umstände anregten. In diesem Sinne ist die Auseinandersetzung um Borodajkewycz im Jahr 1965 als Bruch zu sehen. Auch wenn diese nur vereinzelt in bildungsbürgerlichen Sphären in Wien ausgetragen wurde, löste sie eine öffentliche Diskussion über einen „demokratischen Geschichtsunterricht" aus. Als Fortsetzung dessen lässt sich die kritische mediale Reflexion der institutionalisierten Geschichtsvermittlung aus der "Ohne Maulkorb“-Folge aus dem Jahr 1983 begreifen.

Die Analyse beginnt mit der Berücksichtigung der Bedeutung eines „demokratischen Geschichtsunterrichts" und verknüpft diese mit der von den Alliierten in der unmittelbaren Nachkriegszeit betriebenen Entnazifizierung und Re-Education. Im Anschluss daran werden die beiden ausgewählten Fälle als Beispiele für umstrittene Formen der Geschichtsvermittlung und als Ausdruck der Demokratisierung thematisiert. Schließlich sei an dieser Stelle noch darauf hingewiesen, dass keine allgemeinen Urteile über die Art der Vermittlung des Nationalsozialismus im Geschichtsunterricht getroffen wer-

4 Willi Weinert, Die Entnazifizierung an den österreichischen Hochschulen, in: Sebastian Meiss//Klaus-Dieter Mulley/ Oliver Rathkolb (Hrsg.), Verdrängte Schuld, Verfehlte Sühne. Entnazifizierung in Österreich 1945-1955. Symposion des Instituts für Wissenschaft und Kunst Wien, März 1985, Bad Vöslau 1986, S. 254-269, hier S. 268. 
den können, da es den Geschichtsunterricht oder die Geschichtsvorlesung nicht gab und die inhaltliche Ausrichtung fundamental von der jeweiligen Lehrperson abhing. Nichtsdestotrotz geben die beiden Fallbeispiele Aufschluss über die Formen des Diskurses und dienen damit als Ausgangspunkt für eine aussagekräftige Bestandsaufnahme zum Umgang mit der NS-Vergangenheit.

\section{Umstrittene Lehre. Auf der Suche nach einem „demokratischen Ge- schichtsunterricht"}

\subsection{Enthazifizierung und das demokratische Prinzip}

In der Frage nach der Bedeutung eines "demokratischen Geschichtsunterrichts", wie er in der Demonstration gegen Borodajkewycz am 30. März 1965 gefordert wurde, ist es notwendig, die unmittelbare Nachkriegszeit zu berücksichtigen. In dieser wurde nicht nur die Grundlage für den politischen Neuaufbau des Staates, sondern auch für die Entwicklung eines österreichischen Nationsbewusstseins und einer Identität in Abgrenzung zum völkischen Deutschnationalismus und Nationalsozialismus gelegt. Dafür war es essentiell, die allgemeine, in der Gesellschaft vorherrschende Mentalität und Gesinnung zu verändern. Nach Peter Dinzelbacher ist unter historischer Mentalität „das Ensemble der Weisen und Inhalte des Denkens und Empfindens [zu verstehen], das für ein bestimmtes Kollektiv in einer bestimmten Zeit prägend ist". Die historische Mentalität manifestiere sich in konkreten Handlungen. ${ }^{5}$

Nach der Niederlage im Zweiten Weltkrieg galt es für die Alliierten, die Mentalität als "Inhalte des Denkens und Empfindens" der Österreicher*innen neu zu gestalten, was sie mit den Konzepten der Entnazifizierung und der Re-Education versuchten. Der Begriff "Entnazifizierung" wurde 1945 von den Alliierten eingeführt und bezeichnet sämtliche Bestrebungen, gegen ehemalige Nationalsozialist*innen vorzugehen und sie aus dem öffentlichen Leben zu verdrängen. ${ }^{6}$ Am Beispiel der "Vergangenheitsbewältigung" in der Bundesrepublik Deutschland schildert Dennis Meyer, dass die Entnazifizierung im Sinne einer Demokratisierung der deutschen Bevölkerung betrieben worden sei. Insgesamt gelte sie jedoch als "gescheiterter Versuch einer politischen Massensäuberung". Nichtsdestotrotz sei sie bedeutend für die Verankerung einer Demokratie gewesen.? In Österreich sei die Entnazifizierung laut Dieter Stiefel in den unmittelbaren Nachkriegsjahren ein sehr dominantes und weit verbreitetes Thema gewesen. Fast nichts sei von ihr unberührt geblieben. ${ }^{8}$ Im Gegensatz dazu resümiert Willi Weinert in seiner Analyse der Entnazifizierung an den österreichischen Hochschulen, dass sie in ihrer Fokussierung auf die NSDAP und ihre Organisationen die "geistigen Mittäter" vernachlässigt habe und grundsätzlich als gescheitert betrachtet werden

5 Peter Dinzelbacher, Zur Theorie und Praxis der Mentalitätsgeschichte, in: Peter Dinzelbacher (Hrsg.), Europäische Mentalitätsgeschichte, Stuttgart 1993, S. 15-37, hier S. 21.

6 Thorsten Eitz/Georg Stötzel, Wörterbuch der „Vergangenheitsbewältigung“. Die NS-Vergangenheit im öffentlichen Sprachgebrauch, Hildesheim-Zürich-New York 2007, S. 197.

7 Dennis Meyer, Entnazifizierung, in: Fischer/Lorenz (Hrsg.), Lexikon der „Vergangenheitsbewältigung", S. 20.

8 Dieter Stiefel, Entnazifizierung in Österreich, Wien-München-Zürich 1981, S. 11. 
könne. ${ }^{9}$ Trotzdem sei die Entnazifizierung nicht als vorübergehende Bestrebung, sondern als längerfristiger Prozess anzusehen. ${ }^{10}$ Dies habe vor allem mit den Nachkriegsgenerationen zu tun, was Stiefel in seinem Beitrag aus dem Jahr 1981 folgendermaßen beschreibt: „[...] aber es sind die Nachgeborenen in dem Sinn, daß sie die politischen und sozialen Umwälzungen selbst nicht mehr miterlebt haben, Spuren davon aber immer noch in ihrer eigenen Gesellschaft finden. [...] In diesem Sinn geht die Entnazifizierung bis heute weiter."111 Insofern können öffentliche Infragestellungen von Praktiken der Geschichtsvermittlung als Konfrontationen mit solchen „Spuren“ angesehen werden. Die jüngeren Generationen nehmen dabei jeweils eine zentrale Rolle ein.

Das Konzept der Re-Education ergänzte die Entnazifizierung. Es betonte im Kontext der Nachkriegszeit per definitionem die „politische Umerziehung nach demokratischen Prinzipien“. Die Re-Education umfasste Bestrebungen der Besatzungsmächte mit dem Ziel der "Beseitigung des Faschismus aus dem politischen, kulturellen und ökonomischen Leben und Bewusstsein" der Bevölkerung. ${ }^{12}$ Auf kultureller Ebene ist zum Beispiel die Konfrontation mit den Menschheitsverbrechen in den Konzentrationslagern mittels Dokumentarfilmen in Kinos zu nennen. ${ }^{13}$ Hervorzuheben ist, dass die Umerziehung zwar nicht nur, aber primär von den Alliierten ausging ${ }^{14}$ und deshalb als von oben auferlegte Maßnahme betrachtet werden kann. Des Weiteren gelten sie weitgehend als gescheitert, wobei vor allem die "geistigen Mittäter" vernachlässigt worden seien. ${ }^{15}$ Gleichzeitig ist die Entnazifizierung auch als längerer Prozess anzusehen, der sich über die unmittelbaren Nachkriegsjahre hinaus erstreckt.

Schließlich sind die Entnazifizierung und Re-Education eng mit einem Prozess der Demokratisierung der Gesellschaft verbunden. Dieser Zusammenhang ist für die Frage nach dem „demokratischen Geschichtsunterricht", wie er im Jahr 1965 gefordert wurde, essentiell. Im Sinne einer historischen Mentalität manifestiert sich nämlich der Grad der Entnazifizierung in der sozialen Handlung des Geschichtsunterrichts, und zwar in der Art und Weise, wie die Vergangenheit vermittelt wird. Damit macht der Geschichtsunterricht als soziale Interaktion die Ausprägung der Entnazifizierung erkennbar. So dient die institutionalisierte Form des Lernens und Lehrens von Geschichte als Indikator für die Demokratisierung einer Gesellschaft.

Allgemein kann als Annäherung an die Bedeutung eines „demokratischen Geschichtsunterrichts" ein weites Demokratieverständnis herangezogen werden. In seiner 1916 erstmals erschienenen Publikation bezeichnete der US-amerikanische Philosoph und Pädagoge John Dewey Demokratie als "mehr als eine Regierungform [sic]; sie ist in erster Linie eine Form des Zusammenlebens, der gemeinsamen und miteinander ge-

9 Weinert, Entnazifizierung, S. 268.

10 Stiefel, Entnazifizierung, S. 325.

11 Ebd., S. 12

12 Dennis Meyer, Entnazifizierung, in: Fischer/Lorenz (Hrsg.), Lexikon der "Vergangenheitsbewältigung", S. 21-22.

13 Heiner Roß (Hrsg.), Lernen Sie diskutieren! Re-education durch Film. Strategien der westlichen Alliierten nach 1945, Berlin 2014².

14 Stiefel, Entnazifizierung, S. 332.

15 Weinert, Entnazifizierung, S. 268. 
teilten Erfahrung"16. Diese „miteinander geteilte Erfahrung“ ist gerade für den Umgang mit der (jüngeren) Vergangenheit bedeutsam, da dieser erst ausgehandelt werden muss. In der heute etablierten geschichtsdidaktischen Fachliteratur gibt es ebenfalls Ausführungen zum demokratischen Geschichtsunterricht. Laut Klaus Bergmann handelt es sich dabei um eine Form des Unterrichts, welche an der Herausbildung und Realisierung von demokratischen Grundhaltungen und Abläufen mitwirkt. ${ }^{17}$ Als Maßnahme einer Demokratisierung der Gesellschaft offenbart sich damit die Verbindung zur Entnazifizierung und der Re-Education. Konkret zählt Bergmann unter anderem eine Alltags- und Sozialgeschichte sowie eine Inklusion ausgeschlossener sozialer Gruppen im Sinne einer "Geschichte von unten“ zu seinen Merkmalen. In der Theorie sei demokratischer Geschichtsunterricht als „kommunikative Veranstaltung" gedacht. ${ }^{18}$ Dies bedeute, dass sich die Schüler*innen untereinander sowie mit den Lehrpersonen über "Erfahrung und Wertvorstellungen von Menschen in ihrer Zeit auseinandersetzen, streiten oder verständigen können"19. Besonders bemerkenswert ist dabei, dass der demokratische Geschichtsunterricht per definitionem offen für Streitgespräche, vor allem zwischen Lehrperson und Schüler*innen, angelegt sein sollte. Der Charakter der konfliktreichen Aushandlung eines gemeinsamen Geschichtsverständnisses ist speziell für den Zeitabschnitt der jüngeren Vergangenheit relevant, denn die Epoche bzw. Disziplin der Zeitgeschichte wird bisweilen auch als "Streitgeschichte“ gefasst. ${ }^{20}$ Das darin angedeutete Kontroversitätsgebot - die Möglichkeit, strittige Themen auch kontrovers zu diskutieren - vom Beutelsbacher Konsens aus dem Jahr 1976 ist daher ein Grundprinzip politischer und demokratischer Bildung. ${ }^{21}$ Damit würde ein demokratischer Geschichtsunterricht im Raum der universitären und schulischen Geschichtsvermittlung ein Ringen um die von Dewey angesprochene "miteinander geteilte Erfahrung" eröffnen. Kurz gesagt befürwortet das Konzept des demokratischen Geschichtsunterrichts grundsätzlich eine offene Diskussion und Aushandlung von Geschichte sowie ein Infragestellen von Inhalten und Werten der Geschichtsvermittlung. Anhand zweier konkreter Beispiele wird im Folgenden aufgezeigt, wie sich diese Aushandlung vollzog und wie damit eine Entnazifizierung von unten stattfand.

\subsection{Der Fall Borodajkewycz}

Die Affäre um den Hochschulprofessor Taras Borodajkewycz aus dem Jahr 1965 wurde in der österreichischen Geschichtsschreibung weitgehend aufgearbeitet. Dadurch konnte ihre Bedeutung für die Entwicklung des Umgangs mit der NS-Vergangenheit

16 John Dewey, Demokratie und Erziehung. Eine Einleitung in die philosophische Pädagogik, Weinheim-Basel $2011^{5}$, S. 121.

17 Klaus Bergmann, Geschichtsdidaktik. Beiträge zu einer Theorie historischen Lernens, Schwalbach/Ts. $2000^{2}$, S. 178.

18 Den kommunikativen Aspekt bei der Etablierung einer demokratischen Filmkultur in Roß, Lernen Sie diskutieren!.

19 Bergmann, Geschichtsdidaktik, S. 178.

20 Martin Sabrow/Ralph Jessen/Klaus Große Kracht, Einleitung. Zeitgeschichte als Streitgeschichte, in: Martin Sabrow/Ralph Jessen/Klaus Große Kracht (Hrsg.), Zeitgeschichte als Streitgeschichte. Große Kontroversen nach 1945, München 2003, S. 9-18.

21 Reinhold Gärtner, Basiswissen Politische Bildung, Wien 2016, S. 16-17. 
als "Meilenstein"22 und "Wendepunkt" unterstrichen und eine "langsame Abkehr von der Opferthese ${ }^{\prime 23}$ konstatiert werden. Während eine detaillierte Diskussion der Affäre den Rahmen der Arbeit sprengen würde, sei an dieser Stelle einerseits auf die umfangreiche Forschungsliteratur, insbesondere auf die 2015 erschienene Monografie Rafael Kropiuniggs, verwiesen. ${ }^{24}$ Andererseits soll eine kurze Skizzierung der zentralen Aspekte den notwendigen Rahmen liefern.

Die Kontroverse um den Wirtschaftsgeschichte-Dozenten an der Hochschule für Welthandel nahm mit einer Vorlesungsreihe zur Zwischenkriegszeit und dem Dritten Reich ihren Anfang. Das Unterrichtsministerium hatte zuvor eine zeitgenössische Geschichtsvermittlung als Freifach verlangt. Aufzeichnungen des Wirtschaftsstudenten Ferdinand Lacina (geb. 1942), dem späteren sozialdemokratischen Finanzminister, im Dezember 1961 wurden zum Ausgangspunkt für die kritische Auseinandersetzung. Lacina notierte während der Vorlesungen die für ihn höchst problematischen, doch im Hörsaal nicht einmal ansatzweise kritisch reflektierten Anmerkungen des Professors. Dabei wurden ein latenter Antisemitismus, ein völkischer Deutschnationalismus sowie eine Verherrlichung Adolf Hitlers offen zur Schau gestellt..25 Als "Mitlebender" und Zeitzeuge drückte Borodajkewycz sein „spezifisches Betroffensein durch die Geschichte”26 ohne jeden Vorbehalt aus. Exemplarisch können neben latenten antisemitischen Kommentaren über Hans Kelsen, den maßgeblichen Verfasser der österreichischen Bundesverfassung, auch die von NS-Faszination und Verharmlosung geprägte Erzählung über Adolf Hitler genannt werden. ${ }^{27}$ Durch Lacinas Kontakt zum Juristen und SPÖFunktionär Heinz Fischer (geb. 1938), dem späteren Bundespräsidenten, kam es auf der Grundlage der Vorlesungsaufzeichnungen zu publizistischen Anstrengungen. Fischer veröffentlichte Auszüge der Mitschrift in der sozialdemokratischen „Arbeiter-Zeitung”, was ein erster Versuch war, das Thema in die Öffentlichkeit zu bringen. Daraufhin klagte Borodajkewycz jedoch erfolgreich auf Ehrenbeleidigung, sodass Fischer und ein Redakteur zu einer Geldstrafe verurteilt wurden. ${ }^{28}$

Nachdem die Causa wenige Jahre ruhte, wurde sie am 18. März 1965 erneut aufgegriffen. Dieses Mal war es das relativ junge Medium Fernsehen, das den Ausschlag gab. Die ORF-Satiresendung „Das Zeitventil“ von Gerhard Bronner griff den Inhalt von Lacinas Mitschrift in einem gestellten Interview auf. Ein Schauspieler verkörperte darin den Hochschulprofessor, dessen Originalzitate aus der Vorlesung im Interview verar-

22 Kasemir, Ende, S. 501

23 Lau, Abkehr, S. 28.

24 U. a. Gerard Kasemir, Spätes Ende für „wissenschaftlich“ vorgetragenen Rassismus. Die Borodajkewycz-Affäre 1965, in: Michael Gehler/Hubert Sickinger (Hrsg.), Politische Affären und Skandale in Österreich. Von Mayerling bis Waldheim, Innsbruck 2007, S. 486-501; Arnd Bauerkämper, Das umstrittene Gedächtnis. Die Erinnerung an Nationalsozialismus, Faschismus und Krieg in Europa seit 1945, München 2012, S. 323-324; Rafael Kropiunigg, Eine österreichische Affäre. Der Fall Borodajkewycz, Wien 2015; Lau, Abkehr, S. 28.

25 Kropiunigg, Affäre, S. 11-24.

26 Hans Rothfels, Zeitgeschichte als Aufgabe, in: Vierteljahrshefte für Zeitgeschichte 1 (1953), Heft 1, S. 1-8, hier S. 2.

27 Kropiunigg, Affäre, S. 11-24; siehe auch die Mitschrift der Vorlesung als Ausstellungsexponat im 2018 eröffneten Haus der Geschichte Österreich in Wien.

28 Kropiunigg, Affäre, S. 38. 
beitet und so einem größeren Publikum vermittelt wurden. ${ }^{29}$ Kurze Zeit später bestätigte und wiederholte Borodajkewycz seine antidemokratischen und NS-freundlichen Äußerungen unter dem Beifall von deutschnational gesinnten Studierenden in einer TV-Pressekonferenz. Zudem bekräftigte er auch die Richtigkeit seiner NSDAP-Mitgliedschaft. Als Resultat dieser Medienereignisse konnte eine breitere Masse zum politischen Protest gegen den Hochschulprofessor mobilisiert werden. Am 31. März 1965 kam es in Demonstrationen zum Aufeinanderprallen des rechten und linken Lagers bzw. der Befürworter*innen Borodajkewycz' und seiner Gegner*innen. Erstere ließen den Professor mit Parolen ("Vivat Academia / Vivant Professores”) hochleben, letztere forderten die Bekämpfung des „braunen Gifts”. Zusätzlich fragten sie nach dem in der Einleitung angesprochenen "demokratischen Geschichtsunterricht". ${ }^{0}$ Die gewaltsamen Ausschreitungen gipfelten im Tod Ernst Kirchwegers, einem Antifaschisten und ehemaligen Widerstandskämpfer (1898-1965). ${ }^{31}$ Nach diesen Entwicklungen legte Heinz Fischer Berufung gegen das Ehrenbeleidigungsverfahren ein, woraufhin er und der Redakteur freigesprochen wurden. Erst am 14. Mai 1966 bestrafte der Senat der Hochschule für Welthandel Taras Borodajkewycz mit der zwangsweisen Versetzung in den Ruhestand. ${ }^{32}$

Die Vorlesungsaufzeichnungen von Lacina besitzen als ursprüngliches Medium der Kritik eine besondere Bedeutung für die Geschichte der Zweiten Republik im Allgemeinen und für die Aufarbeitung der NS-Vergangenheit im Speziellen. Dies unterstreicht nicht zuletzt die Tatsache, dass sie als Objekt im 2018 eröffneten Haus der Geschichte Österreich ausgestellt sind. Im Themenabschnitt „Diktatur, NS-Terror, Erinnerung” nehmen sie neben dem vergleichsweise monumentalen "Waldheim-Pferd" von Alfred Hrdlicka einen zentralen Platz im österreichischen Gedächtnis ein. In der historischen Aufarbeitung des Skandals ist jedoch auffällig, dass die Parolen bei den Demonstrationen vom März 1965 als Ausdruck der politischen (Protest-)Kultur weniger im Vordergrund standen. Eine genauere Betrachtung dieser - allen voran der Frage nach einem „demokratischen Geschichtsunterricht" - macht jedoch einen Prozess der Demokratisierung der Gesellschaft als Forderung von Teilen aus der Gesellschaft erfassbar. Insofern ist der Fall Borodajkewycz als Vorläufer der 68er-Bewegung zu betrachten. Laut Wernecke habe sich in der bundesdeutschen Gesellschaft seit den späten 1950er-Jahren langsam ein Trend zu einer Liberalisierung und Demokratisierung abgezeichnet. Dieser sei auch mit einer Forderung nach Entnazifizierung an den Hochschulen und konkreten Aufforderungen sowie publizistischen Tätigkeiten von Studierenden an mehreren deutschen Universitäten im Studienjahr 1964/1965 verbunden gewesen. ${ }^{33}$

29 Ebd., S. 44; siehe dazu das Material in der ORF TVthek: Die Originalsendung aus dem Jahr 1965 wurde nicht archiviert, allerdings erfolgte 1994 eine erneute Nachstellung mit einer Einführung von Gerhard Bronner, siehe: ORF TVthek, Fiktives Interview mit Taras Borodajkewycz, 23.12.1994, [https://tvthek.orf.at/history/Der-FallBorodajkewycz/9427047/Fiktives-Interview-mit-Taras-Borodajkewycz/9462767], eingesehen 1.3.2020.

30 ORF TVthek, Demo gegen Taras Borodajkewycz, 31.3.1965, [https://tvthek.orf.at/history/Der-FallBorodajkewycz/9427047/Demo-gegen-Taras-Borodajkewycz/9440238], eingesehen 1.3.2020.

31 Bauerkämper, Gedächtnis, S. 323-324; allgemein zum Fall Borodajkewycz: ORF TVthek, Der Fall Borodajkewycz o. D. [https://tvthek.orf.at/history/Der-Fall-Borodajkewycz/9427047], eingesehen 01.03.2020.

32 Kasemir, Spätes Ende, S. 500.

33 Klaus Wernecke, 1968, in: Fischer/Lorenz (Hrsg.), Lexikon der „Vergangenheitsbewältigung“, S. 188-189. 
Laut Kropiunigg habe die Affäre in Österreich eine "versäumte Entnazifizierung"34 offenbart, wobei die Entnazifizierung hier als der Prozess von oben verstanden werden muss. Zu den wesentlichen Schauplätzen in der Causa gehörten die Vorlesung bzw. die Hochschule, die Medien und die Straße als politisches Konfliktfeld, ${ }^{35}$ aber nicht primär die Politik. Ursprünglich initiiert von Studierenden und Akteur*innen der jüngeren Generation wie Lacina oder dem Juristen und SPÖ-Funktionär Fischer, kann sie damit als eine Bewegung von unten angesehen werden. Im Kern richtete sie sich gegen ein nationalsozialistisches, antisemitisches und völkisch deutschnationales Geschichtsbild im Rahmen der zeithistorischen Vermittlung an der Hochschule für Welthandel. Es sei dabei für Lehrende möglich gewesen, den Studierenden und damit der Bildungselite weitgehend ungehindert und unhinterfragt antidemokratische und deutschnationale Anschauungen zu vermitteln. ${ }^{36}$ In Anlehnung an Stiefels Wortlaut fanden einzelne Akteur*innen somit „Spuren“37 der nationalsozialistischen Vergangenheit in ihrer Lebenswelt wieder, äußerten ihre Kritik an der antidemokratischen Einstellung und gingen mit demokratischen Mitteln dagegen vor. Diese beinhalteten die friedlichen Mittel der Erzeugung von öffentlichem, außerparlamentarischem Diskurs und Protesten. Insofern war eine kritisch eingestellte Minderheit um eine Konfrontation mit Relikten nationalsozialistischer Anschauungen bemüht. Dies kommt einer Entnazifizierung von unten sowie einer Demokratisierung von unten gleich. So war dies gleichzeitig ein Versuch der Umsetzung eines demokratischen Geschichtsunterrichts, der mit der Forderung desselben einherging. Im offenen Streit zwischen Lehrenden (Borodajkewycz) und Studierenden (Lacina u. a.) bzw. einer kritischen Öffentlichkeit fand eine Auseinandersetzung gemäß der Definition Bergmanns statt: ein kontroverser Umgang mit "Erfahrung und Wertvorstellungen von Menschen in ihrer Zeit"38.

Wenngleich das Ergebnis der Affäre keinen radikalen Wandel bewirkte, ${ }^{39}$ so war es dennoch ein folgenreicher Bruch in der österreichischen Nachkriegsgesellschaft. Dieser zeigte auf, dass die öffentliche Infragestellung von Lehrpraktiken und -inhalten in einer Demokratie möglich sein musste. Die Vermittlung der NS-Vergangenheit im Rahmen der Zeitgeschichte als "Epoche der Mitlebenden"40 und der damit einhergehenden Herausforderungen für die Rolle der Vermittlungsperson blieben nicht mehr länger unhinterfragt. Deshalb kann der Fall Borodajkewycz mit der Forderung eines "demokratischen Geschichtsunterrichts" als ein Akt studentischen bzw. zivilgesellschaftlichen Ungehorsams angesehen werden.

\subsection{Hans-Ulrich Rudel als "Vorbild" und "Held"}

Als Heinz Fischer im Jahr 1985 - nun in seiner Funktion als SPÖ-Wissenschaftsminister in der Regierung Sinowatz - ein Symposion über die Entnazifizierung in Österreich eröff-

\footnotetext{
Kropiunigg, Affäre, S. 10.

Ebd., S. 89

Ebd., S. 11

Stiefel, Entnazifizierung, S. 12.

Bergmann, Geschichtsdidaktik, S. 178.

Kropiunigg, Affäre, S. 84

Rothfels, Zeitgeschichte als Aufgabe, S. 2.
} 
nete, machte er folgende Feststellung: „Ich bin fest überzeugt, daß die Relikte des Verständnisses für nationalsozialistische Gesinnung oder auch nur einzelne Elemente derselben in den letzten 20 Jahren wesentlich geringer geworden sind." ${ }^{\prime 41}$ Die Tagung über die Entnazifizierung in Österreich im März 1985 kam zu einem besonderen Zeitpunkt: einerseits ein Jahr vor der Waldheim-Affäre, die zweifelsfrei den markantesten Wendepunkt im Umgang Österreichs mit der NS-Vergangenheit darstellte, und andererseits zwanzig Jahre nach dem Fall Borodajkewycz. So liefert die Einschätzung Fischers über den Rückgang von Tendenzen der NS-Ideologie eine interessante Zwischenbilanz. Bei der Untersuchung der institutionalisierten Vermittlung von Zeitgeschichte in Gesellschaft, (Hoch-)Schule und Unterricht bietet sich mit der Folge "Geschichtsunterricht" der ORF-Fernsehsendung „Ohne Maulkorb” aus dem Jahr 1983 eine aufschlussreiche Quelle für diese Zeit an.

Die Folge verdeutlicht eine Fortsetzung des Diskurses und der Aushandlung von Geschichte als Entnazifizierung von unten sowie einem "demokratischen Geschichtsunterricht". Mit der Zäsur bei Borodajkewycz wurde gezeigt, dass Lehrende in ihren Machtpositionen unter Berufung auf demokratische Prinzipien öffentlich hinterfragt werden können. Was im Jahr 1965 noch aufgrund der Unantastbarkeit von Lehrenden eine umfassende gesellschaftliche Bewegung benötigte und gewaltsame Reaktionen auslöste, konnte im Jahr 1983 ohne großen Widerstand der Gegenseite auch im kleineren Rahmen artikuliert werden. Dies kommt durch eine genauere Analyse der Sendung zum Vorschein.

Die Folge des Jugendmagazinformates „Ohne Maulkorb“ wurde am 18. Februar 1983 ausgestrahlt und war eine Bestandsaufnahme des Geschichtsunterrichts in einer Wiener Schule. Der Beitrag dauert ca. 24 Minuten, lässt Jugendliche in ihrer Lebenswelt Schule sowie auch Expert*innen aus Wissenschaft und Politik zu Wort kommen. In seiner Anmoderation nennt Peter Resetarits (geb. 1960) 50-jährige Jubiläen und bedeutende Jahrestage für die österreichische Geschichte, so etwa die Ausschaltung des Parlaments am 4. März 1933, als Anlass für die Sendung. ${ }^{42}$ Der mittlerweile langjährige ORF-Journalist spricht dabei bereits eine Vernachlässigung zeithistorischer Themen im Unterricht an. Zudem ist Peter Resetarits neben dem Aspekt des erinnerungskulturellen Agenda-Settings des Jubiläums hier nennenswert, weil er eine Verknüpfung zur formalen Ebene ermöglicht. Sein Bruder, Willi Resetarits (geb. 1948), untermalt mit der Musik seiner Band Schmetterlinge („Faschismuslied des Geschichtslehrers“) die inhaltliche Ebene. Die mediale Inszenierung lässt daher gemäß der jugendlichen Zielgruppe eine Ausrichtung an einer kritischen und alternativen Subkultur erkennen.

Im Laufe der Sendung erzählen Schüler*innen davon, was sie im Fach Geschichte lernen und wie sie persönlich dazu stehen. Zum Beispiel sagt eine Jugendliche: „In den Geschichtsbüchern steht wie es war, das sind Tatsachen, die muss der Schüler akzeptieren. Und die eigene Meinung, die wird eigentlich unterdrückt, weil man ja gleich

41 Heinz Fischer, Eröffnung des Symposions Entnazifizierung in Österreich, in: Meissl/Mulley/Rathkolb (Hrsg.), Verdrängte Schuld, Verfehlte Sühne, S. 13.

42 Michael Kreissl, ,Geschichtsunterricht”, in: Ohne Maulkorb, Österreichischer Rundfunk, 18.2.1983, 00:00-01:00 min. 
zum nächsten Stoff übergehen muss." ${ }^{43}$ Neben der Behandlung von Kriegen, Schlachten und Königen würde es im Geschichtsunterricht hauptsächlich um das Auswendiglernen von Jahreszahlen gehen. Des Weiteren kommt die fehlende Berücksichtigung der jüngeren Vergangenheit, beispielsweise der Jahre 1933/34, der Verbrechen des Faschismus oder des Widerstandes dagegen, zur Sprache. Dabei wird die Rolle der Lehrperson adressiert. Konkret stellt ein Redakteur die Frage, ob es „auch besonders extreme Beispiele von Lehrern, deren Einstellung vielleicht dann hin und wieder an der Grenze der Demokratie zu sein scheint", gebe. Daraufhin antwortet ein Vertreter der „Schülerzeitungsgewerkschaft": „Nein, nicht nur an der Grenze, sondern auch darüber hinaus." Als Beispiel dafür nennt er einen Professor aus einem Bundesgymnasium, der seiner Klasse nahegelegt habe, sie sollten sich an „Oberst Rudel ein Beispiel nehmen” und diesen als "Vorbild" betrachten. Dies habe sich so zugetragen, dass der Lehrer in einer Diskussion Hans-Ulrich Rudel als "Helden“ bezeichnet habe, der "hinter den feindlichen Linien gelandet ist, um seine Kameraden zu retten und mit Schulterschuss durch die eiskalte Wolga geschwommen ist. Kein Wort davon, dass das eine der schillerndsten Figuren der neofaschistischen Szene in Deutschland ist".44

Zur besseren Einordnung dieser Aussagen des Schülers sowie der Lehrperson ist der AHS-Oberstufenlehrplan dieser Zeit heranzuziehen. Laut dem Historiker Heinz P. Wassermann reichte die damals letzte größere Reform in das Jahr 1970 zurück. Speziell für die didaktischen Grundsätze hebt Wassermann die neue Aufforderung aus dem Lehrplan hervor, dass in "allen Klassen [...] bei sich bietendem Anlass Probleme des Zeitgeschehens in objektiver Weise" zu behandeln seien. ${ }^{45}$ In dieser Reform hätten sich die zeitlichen Grenzen für die thematische Abhandlung in den einzelnen Jahrgängen ebenfalls geändert. So sei in der achten Klasse der Zeitraum vom Beginn des Ersten Weltkrieges bis zur Gegenwart vorgesehen. Zumindest in der Theorie bedeutete dies eine stärkere Beachtung der jüngeren Vergangenheit. Darüber hinaus belegt der Lehrplan die weitgehende Ausklammerung des Holocaust. ${ }^{46}$ Einen ähnlichen Befund liefern Schulbuchanalysen von österreichischen Lehrwerken. Demnach habe es bis in die 1980er-Jahre wenig oder gar keine Berücksichtigung des Themas der (österreichischen) NS-Täterschaft gegeben. Die Darstellung der Zeit des Zweiten Weltkrieges sei bis auf wenige Ausnahmen vom Opfermythos gekennzeichnet gewesen. Österreichische Soldaten der deutschen Wehrmacht seien auch nicht in Bezug auf Kriegsverbrechen behandelt worden. ${ }^{47}$ Wenngleich der Lehrplan als normative Grundlage mit der Aufforderung zur Objektivität oder der Hinwendung zur Zeitgeschichte in der achten Klasse als progressiv erscheint, offenbart die Schulbuchanalyse zentrale Leerstellen.

\footnotetext{
43 Kreissl, "Geschichtsunterricht”, 03:38-03:48 min.

44 Ebd., 15:48-16:42 min.

45 Heinz P. Wassermann, Verfälschte Geschichte im Unterricht. Nationalsozialismus und Österreich nach 1945, Innsbruck-Wien 2004, S. 46.

46 Wassermann, Verfälschte Geschichte, S. 47.

47 David Hasenauer, "Vergangenheitsbewältigung“ in österreichischen Schulbüchern seit 1955. Die Darstellung von "Anschluss", NS-Verbrechen, österreichischem Widerstand und (Nach-)Kriegserinnerungen bis heute, Dipl. Innsbruck 2015, S. 185
} 
Von einer Objektivität, geschweige denn einer Anerkennung der Kontroversität der Themen, ist beim geschilderten Vorfall der Sendung "Ohne Maulkorb“ keinesfalls zu sprechen. Der Beutelsbacher Konsens aus dem Jahr 1976, der neben dem Kontroversitätsgebot das fundamentale Indoktrinationsverbot enthält, ${ }^{48}$ ist bei der Lehrperson im Jahr 1983 nicht ansatzweise zu erkennen. Vielmehr kommt die Verehrung und die Bewunderung eines von der NS-Propaganda zur Legende hochstilisierten Wehrmachts-Kampffliegers, eines späteren NS-Fluchthelfers und weiterhin aktiven Nationalsozialisten ${ }^{49}$ zum Ausdruck. Diese lehnt sich dabei in ihren Grundzügen an den kontroversiellen Diskurs der (Neu-)Bewertung der Wehrmacht ab den 1990er-Jahren an. ${ }^{50}$ Sie geht aber auch darüber hinaus, da der Lehrer - wie der Schüler andeutet - keine kritische Reflexion des in neonazistischen Organisationen tätigen Mannes vornimmt. In der Einschätzung des Schülers, dass sich der Lehrer "an der Grenze der Demokratie“ bzw. „darüber hinaus" bewege, kommen die öffentliche Infragestellung der Geschichtsinterpretation und damit auch der Ansatz eines demokratischen Geschichtsunterrichts zum Ausdruck.

Gleichermaßen steht dieses Hinterfragen der Gesinnung des Lehrers für eine Fortführung der Entnazifizierung von unten, wie sie bereits mit der Kritik an Taras Borodajkewycz im Jahr 1965 zum Vorschein kam. Da der Lehrer von der Redaktion mit der Bitte um eine Stellungnahme ohne Erfolg kontaktiert worden sei, konnte im Falle des Jahres 1983 kein Gespräch mit dem Lehrer stattfinden. ${ }^{51}$ Nichtsdestotrotz macht der Vorfall auch den Prozess einer Demokratisierung sichtbar: Die Schüler*innen bekamen im Format des Fernseh-Jugendmagazins eine mediale Bühne, die ihre Stimme aufzeigte, andere Geschichtslehrer*innen sowie Expert*innen aus der Geschichtswissenschaft einbezog und den Diskurs mit politischen Verantwortlichen aus dem Unterrichtsministerium ermöglichte. Damit konnte letztlich eine Multiperspektivität im gesellschaftlichen Dialog hergestellt und eine eminent demokratische, das heißt eine offene, sachliche, kritische und vor allem gewaltfreie Auseinandersetzung gewährleistet werden. Somit veranschaulicht die "Ohne Maulkorb“-Folge eine Fortführung der Entnazifizierung und Demokratisierung von unten und steht als mediale Repräsentation selbst für eine „kommunikative Veranstaltung ${ }^{\prime \prime 2}$ im Sinne eines demokratischen Geschichtsunterrichts.

\section{Schluss und Ausblick}

Zusammenfassend kann festgehalten werden, dass die beiden Fallbeispiele Aufschluss über die Diskurse des Umgangs mit der NS-Vergangenheit in Bildung, (Hoch-)Schule und Gesellschaft geben. Der Umgang mit dem Nationalsozialismus darin gestaltete

\footnotetext{
48 Gärtner, Basiswissen, S. 16-17.

49 Sönke Neitzel, Rudel, Hans-Ulrich, in: Deutsche Biographie, o. D., [https://www.deutsche-biographie.de/ gnd1 18603655.html\#ndbcontent], eingesehen 20.04.2019; Der Spiegel, 03.01.1983, S. 65-66.

50 Lena Knäpple, Wehrmachtsausstellung, in: Fischer/Lorenz (Hrsg.), Lexikon der "Vergangenheitsbewältigung", S. 312-314.

51 Kreissl, "Geschichtsunterricht", 16:42-16:53 min.

52 Bergmann, Geschichtsdidaktik, S. 178.
} 
sich ähnlich problematisch und herausfordernd wie in anderen politischen, kulturellen und sozialen Bereichen und musste erst in demokratischen Prozessen ausgehandelt werden. Die Zeitgeschichte als jüngere Vergangenheit wurde im Rahmen der universitären Lehre und des schulischen Geschichtsunterrichts auf unterschiedliche, teils besonders kontroverse Art und Weise vermittelt. Wie die analysierten Beispiele zeigen, entsprechen die Kritik und die öffentliche Infragestellung von als undemokratisch empfundenen Praktiken den gesellschaftlichen Prozessen einer Entnazifizierung und Demokratisierung von unten. Im Verständnis von John Dewey ist Demokratie dabei eine Art der Koexistenz und der kollektiven Erfahrung, die es in der Gesellschaft auszuhandeln gilt.

Unter dem Eindruck aktueller Ereignisse und Debatten ist zu konstatieren, dass dieser Prozess der Aushandlung auch im Jahr 2020 andauert. So steht die Causa um den FPÖnahen Professor Lothar Höbelt vom Institut für Geschichte der Universität Wien ${ }^{53}$ in einer Tradition mit den beschriebenen Fallbeispielen. Die Nähe eines Dozenten zum Rechtsextremismus, Deutschnationalismus sowie Geschichtsrevisionismus erinnert einmal mehr an die Ereignisse, die Kritik und die Proteste aus dem Jahr 1965 sowie an die Frage aus dem Anti-Borodajkewycz-Lager: „Wo bleibt der demokratische Geschichtsunterricht?" In der Begegnung damit sind demokratische Mittel wie "die Kraft zur Reflexion, zur Selbstbestimmung, zum Nicht-Mitmachen" ${ }^{45}$ von essentieller Bedeutung. Nicht zuletzt besteht genau darin der Kern einer demokratischen Auseinandersetzung über die Gestaltung einer Erziehung nach Auschwitz.

\section{Literatur und Quellen}

APA Picturedesk, 19650330_PD0002 (RM), 30.03.1965, [https://www.picturedesk.com/ bild-disp/search/search.action?ts=1555661039786\&data=eyJicil6WylwOjlxOmJvcm9kYWprZXd5Y3oiXSwicnMiOjEsInNOIjoiUVVFUllfU1RSSU5HliwiaXNwljpmYWxzZ SwiaXNzljpmYWxzZSwic29ydCI6MCwibnJwljo1MCwicG4iOjF9\&searchID=20d2da56eb13-4579-8a9b-78290ce13bd8], eingesehen 19.04.2019.

Adorno, Theodor W., Erziehung nach Auschwitz, in: Gerd Kadelbach (Hrsg.), Theodor W. Adorno. Erziehung zur Mündigkeit. Vorträge und Gespräche mit Hellmut Becker 1959-1969, Frankfurt a. M. 2008, S. 88-104.

Bauerkämper, Arnd, Das umstrittene Gedächtnis. Die Erinnerung an Nationalsozialismus, Faschismus und Krieg in Europa seit 1945, München 2012.

Bergmann, Klaus, Geschichtsdidaktik. Beiträge zu einer Theorie historischen Lernens, Schwalbach/Ts. 2000².

Der Spiegel, 03.01.1983, S. 65-66.

53 O. A., Lothar Höbelt, Professor Einzelfall, in: der Standard.at, 22.1.2020, [https://www.derstandard.at/story/ 2000113531274/lothar-hoebelt-professor-einzelfall], eingesehen 1.3.2020.

54 Adorno, Erziehung, S. 93. 
Dewey, John, Demokratie und Erziehung. Eine Einleitung in die philosophische Pädagogik, Weinheim-Basel $2011^{5}$.

Dinzelbacher, Peter, Zur Theorie und Praxis der Mentalitätsgeschichte, in: Peter Dinzelbacher (Hrsg.), Europäische Mentalitätsgeschichte, Stuttgart 1993, S. 15-37.

Eitz, Thorsten/Stötzel, Georg, Wörterbuch der „Vergangenheitsbewältigung”. Die NSVergangenheit im öffentlichen Sprachgebrauch, Hildesheim-Zürich-New York 2007.

Fischer, Heinz, Eröffnung des Symposions Entnazifizierung in Österreich, in: Sebastian Meissl/Klaus-Dieter Mulley/Oliver Rathkolb (Hrsg.), Verdrängte Schuld, Verfehlte Sühne. Entnazifizierung in Österreich 1945-1955. Symposion des Instituts für Wissenschaft und Kunst Wien, März 1985, Bad Vöslau 1986, S. 9-13.

Gärtner, Reinhold, Basiswissen Politische Bildung, Wien 2016.

Hasenauer, David, „Vergangenheitsbewältigung” in österreichischen Schulbüchern seit 1955. Die Darstellung von „Anschluss“, NS-Verbrechen, Österreichischem Widerstand und (Nach)Kriegserinnerungen bis heute, Dipl. Innsbruck 2015.

Höft, Andrea, Nationalsozialismus im Schulunterricht, in: Torben Fischer/Matthias Lorenz (Hrsg.), Lexikon der "Vergangenheitsbewältigung" in Deutschland. Debatten- und Diskursgeschichte des Nationalsozialismus nach 1945, Berlin 2015, S. 182-186.

Kasemir, Gerard, Spätes Ende für "wissenschaftlich“ vorgetragenen Rassismus. Die Borodajkewycz-Affäre 1965, in: Michael Gehler/Hubert Sickinger (Hrsg.), Politische Affären und Skandale in Österreich. Von Mayerling bis Waldheim, Innsbruck 2007, S. 486-501.

Knäpple, Lena, Wehrmachtsausstellung, in: Fischer/Lorenz (Hrsg.), Lexikon der „Vergangenheitsbewältigung", S. 312-314.

Kreissl, Michael, "Geschichtsunterricht", in: Ohne Maulkorb, Österreichischer Rundfunk, 18.02.1983.

Kropiunigg, Rafael, Eine österreichische Affäre. Der Fall Borodajkewycz, Wien 2015.

Lau, Johannes, Langsame Abkehr von der Opferthese, in: Der Standard, 03.10.2018.

Meyer, Dennis, Entnazifizierung, in: Fischer/Lorenz (Hrsg.), Lexikon der „Vergangenheitsbewältigung", S. 20-21.

Ders., Reeducation, in: Fischer/Lorenz (Hrsg.), Lexikon der "Vergangenheitsbewältigung", S. 21-23.

Neitzel, Sönke, Rudel, Hans-Ulrich, in: Deutsche Biographie, o.D., [https://www.deutsche-biographie.de/gnd118603655.html\#ndbcontent], eingesehen 20.04.2019.

o. A., Lothar Höbelt, Professor Einzelfall, in: der Standard.at, 22.01.2020, [https://www. derstandard.at/story/2000113531274/lothar-hoebelt-professor-einzelfall], eingesehen 01.03.2020. 
ORF TVthek, Demo gegen Taras Borodajkewycz, 31.03.1965, [https://tvthek.orf.at/history/Der-Fall-Borodajkewycz/9427047/Demo-gegen-Taras-Borodajkewycz/9440238], eingesehen 01.03.2020.

ORF-TVthek, Fiktives Interview mit Taras Borodajkewycz, 23.12.1994, [https://tvthek. orf.at/history/Der-Fall-Borodajkewycz/9427047/Fiktives-Interview-mit-Taras-Borodajkewycz/9462767], eingesehen 01.03.2020.

ORF TVthek, Der Fall Borodajkewycz, o. D. [https://tvthek.orf.at/history/Der-Fall-Borodajkewycz/9427047], eingesehen 01.03.2020.

Roß, Heiner (Hrsg.), Lernen Sie diskutieren! Re-education durch Film. Strategien der westlichen Alliierten nach 1945, Berlin 2014².

Rothfels, Hans, Zeitgeschichte als Aufgabe, in: Vierteljahrshefte für Zeitgeschichte 1 (1953), Heft 1, S. 1-8.

Sabrow, Martin/Jessen, Ralph/Große Kracht, Klaus, Einleitung. Zeitgeschichte als Streitgeschichte, in: Martin Sabrow/Ralph Jessen/Klaus Große Kracht (Hrsg.), Zeitgeschichte als Streitgeschichte. Große Kontroversen nach 1945, München 2003, S. 9-18.

Stiefel, Dieter, Entnazifizierung in Österreich, Wien-München-Zürich 1981.

Wassermann, Heinz P., Verfälschte Geschichte im Unterricht. Nationalsozialismus und Österreich nach 1945, Innsbruck-Wien 2004.

Weinert, Willi, Die Entnazifizierung an den österreichischen Hochschulen, in: Meissl/ Mulley/Rathkolb (Hrsg.), Verdrängte Schuld, Verfehlte Sühne, S. 254-269.

Wernecke, Klaus, 1968, in: Fischer/Lorenz (Hrsg.), Lexikon der „Vergangenheitsbewältigung", S. 188-193.

Benedikt Kapferer ist Mitarbeiter am Institut für Zeitgeschichte der Universität Innsbruck. Im Dezember 2019 schloss er sein Lehramtsstudium der Fächer Geschichte, Sozialkunde und Politische Bildung und Englisch an der Universität Innsbruck ab. benedikt.kapferer@uibk.ac.at

\section{Zitation dieses Beitrages}

Benedikt Kapferer, „Wo bleibt der demokratische Geschichtsunterricht?” Der Umgang mit der NS-Vergangenheit in Schulbildung und Gesellschaft am Beispiel von Taras Borodajkewycz und Hans-Ulrich Rudel, in: historia.scribere 12 (2020), S. 231-245, [http:// historia.scribere.at], eingesehen 15.6.2020 (=aktuelles Datum).

Creative Commons Licences 3.0 Österreich unter Wahrung der Urheberrechte der Autorlnnen. 
